\title{
Combined effects of disease management and food insecurity on physical and mental health in Korean adults
}

\author{
Hyun Ja Kim ${ }^{1, \ddagger}$ and Kirang Kim ${ }^{2, *}$ \\ 'Department of Food and Nutrition, Gangneung-Wonju National University, Gangneung, Gangwon-do, Republic of \\ Korea: ${ }^{2}$ Department of Food Science and Nutrition, Dankook University, Cheonan 3111 6, Republic of Korea
}

Submitted 26 December 2018: Final revision received 26 June 2019: Accepted 9 July 2019: First published online 20 November 2019

\begin{abstract}
Objective: The present study aimed to examine the combined effects of disease management and food insecurity on physical and mental health in a representative Korean population.

Design: A cross-sectional study.

Setting: Data from the Korea National Health and Nutrition Examination Survey (KNHANES) 2012-2015.

Participants: Adults aged $\geq 30$ years ( $n$ 17 934) who participated in the KNHANES. Results: Among health-care factors, unmet health-care needs and mental health counselling were different by food insecurity status, with a higher prevalence in adults with food insecurity. The prevalence of underweight was higher in men with food insecurity (5.9\%), whereas the prevalence of obesity was higher in women with food insecurity (37.4\%), than that in men and women with food security. Food insecurity was associated with a high risk of all mental health outcomes. For the combined effects of disease management and food insecurity, unmet health-care needs was related to increased risk of obesity for food-insecure men $\left(P_{\text {interaction }}=0.029\right)$ and lack of participation in nutrition education or counselling was related to increased risk of obesity for food-insecure women $\left(P_{\text {interaction }}=0 \cdot 010\right)$. In addition, higher unmet health-care needs in adults with food insecurity was related to higher risk of mental health outcomes.

Conclusions: Unmet health-care needs may exacerbate obesity for food-insecure men and mental health problems for both food-insecure men and women. In addition, lack of participation in nutrition education or counselling may exacerbate the obesity for food-insecure women.
\end{abstract}

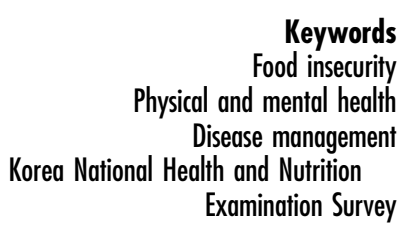

Food security is defined as the access to enough food by all people, at all times, for an active and healthy life; food insecurity occurs when individuals or households cannot meet adequate and safe food and nutritional needs in socially acceptable ways ${ }^{(1)}$. Although food insecurity is associated with reduced nutrient and energy intakes, it has also been associated with chronic diseases such as obesity, hypertension, diabetes and hyperlipidaemia ${ }^{(2,3)}$. In addition, food insecurity is known to contribute to psychological health, including depression symptoms and even suicide ideation, through environmental adversity, disadvantage and stressful events in individuals with low socio-economic status ${ }^{(4-7)}$. A previous cross-sectional

*The original version of this article contained incorrect indexing information. A notice detailing this has been published and the error rectified in the online PDF and HTML copies. study reported that food insecurity was more prevalent in adults with mood disorders and associated with worsening of their mania symptoms ${ }^{(8)}$.

Adults with food insecurity are known to be at risk of physical inactivity, cigarette smoking, drinking, skipping breakfast and inadequate micronutrient intakes ${ }^{(2,9,10)}$, which are potentially detrimental to their health conditions. Recent studies reported that individuals with food insecurity are more likely to be non-compliant to medications and have limitations in achieving a healthy lifestyle due to financial burden ${ }^{(11-14)}$. Particularly, the results showed that women were more likely to report cost-related medication underuse than men ${ }^{(11,13)}$. The vulnerability of food insecurity could lead individuals to pay little attention to disease management, such as taking medications in a timely manner and receiving education or counselling related to 
diseases $^{(12,14-16)}$. The poor management of conditions among adults with food insecurity with one or more chronic illnesses would exacerbate their illnesses. Given the importance of health management on food insecurity and health outcomes, studies on their relationship in a nationally representative sample are limited.

Despite increased national food availability and purchasing power caused by rapid economic growth, food insecurity in South Korea is still high among lower socioeconomic households, accounting for $54 \%$ in the lowest household income group ${ }^{(17)}$. Recently, differences in the quantity and quality of dietary intake based on socioeconomic status have widened over time, leading to nutrition and health inequalities ${ }^{(18-21)}$. The Korean government has planned to increase the proportion of households with food security as one of the objectives of the Health Plan 2020, and monitored its status and developed intervention programmes ${ }^{(22)}$. Therefore, the objectives of the present study were to examine the combined effects of food insecurity and disease management on physical and mental health to explore whether lack of disease management may exacerbate the physical or mental health of adults with food insecurity, and to investigate whether there is a gender difference in these effects, among the representative Korean population using data from the Korea National Health and Nutrition Examination Survey (KNHANES).

\section{Methods}

\section{Data}

The present study was based on data from the consecutive KNHANES conducted from 2012 to 2015 by the Korea Centers for Disease Control and Prevention. These data were obtained from the KNHANES website (https:// knhanes.cdc.go.kr/knhanes/sub03/sub03_02_02.do). The KNHANES is a nationwide cross-sectional survey initiated in 1998 to examine the health and nutritional status of the non-institutionalized Korean population. Using a stratified and multistage clustered probability sampling method, 31006 representative Koreans aged $\geq 1$ year have participated in the KNHANES from 2012 to 2015. Among 18170 adults aged $\geq 30$ years who participated in all health interviews, examinations and nutrition surveys, 17934 adults (7247 men and 10687 women) who completed the food insecurity questionnaire were included in the present analysis. Health interviews and examinations were conducted by trained medical staff and interviewers in mobile examination centres. The health interview provided detailed information on socio-economic characteristics (e.g. sex, age, education, marital status, household income, area of residence, recipients of basic livelihood security), health-related behaviours (e.g. smoking, drinking, activity), disease management (e.g. taking medications, trying to control weight, health examination, mental health counselling, nutrition education or counselling, unmet health-care needs) and mental health (e.g. perceived stress, depression experience, suicidal ideation). Anthropometrical, biochemical and clinical profiles were measured during the health examination survey.

\section{Disease management-related factors}

Questions, answerable by 'yes' or 'no', on the intake of medications for hypertension, diabetes or hypercholesterolaemia were asked to participants diagnosed previously with these diseases. Respondents who answered 'trying to lose weight', 'trying to maintain weight' or 'trying to gain weight' to 'Have you ever tried to control your weight during the last year?' were classified into the category of 'trying to control weight'. We made the remaining response ('never tried') a reference category to calculate the OR. Information on health examination, mental health counselling, nutrition education or counselling and unmet health-care needs was obtained using a 'yes' or 'no' answer to 'Have you received medical examination for health in the past 2 years?', 'Have you ever received counselling through visit, telephone, Internet or other means due to psychological problem in the last year?', 'Have you ever received nutrition education or counselling in the last year?' and 'Have you ever been unable to go to a hospital/clinic (excluding dental clinics) in the past year even though you needed to do so?'.

\section{Physical bealth outcomes}

Height to the nearest $0 \cdot 1 \mathrm{~cm}$ was measured using a portable stadiometer. Weight to the nearest $0 \cdot 1 \mathrm{~kg}$ was measured using a portable electronic scale. BMI was calculated as the ratio of weight to height squared $\left(\mathrm{kg} / \mathrm{m}^{2}\right)$. Obesity and underweight were defined as $\mathrm{BMI} \geq 25.0 \mathrm{~kg} / \mathrm{m}^{2}$ and $\mathrm{BMI}<18.5 \mathrm{~kg} / \mathrm{m}^{2}$, respectively, based on the redefined criteria of the WHO for obesity in the Asia-Pacific region ${ }^{(23)}$. Hypertension was defined as mean systolic blood pressure and/or diastolic blood pressure of $\geq 140 / 90 \mathrm{mmHg}$ or the current use of antihypertensive agents based on the criteria in the seventh report of the Joint National Committee ${ }^{(24)}$. Based on the criteria of the $\mathrm{WHO}^{(25)}$, diabetes was defined as a fasting plasma glucose of $\geq 126 \mathrm{mg} / \mathrm{dl}$, a previous diagnosis of diabetes by a physician or the current use of antidiabetic agents or insulin. Hypercholesterolaemia was defined as a plasma total cholesterol of $\geq 240 \mathrm{mg} / \mathrm{dl}$ or the current use of cholesterol-lowering agents based on the guidelines for cholesterol of the National Cholesterol Education Program ${ }^{(26)}$.

\section{Mental bealth outcomes}

Self-administered questionnaires were used to obtain information on mental health. The questions for mental health outcomes were developed based on the questionnaires of the Canadian Community Health Survey (CCHS) ${ }^{(27,28)}$. The participants selected their stress status using a four-point scale ('extremely high', 'high', 'a little bit', 'almost not') to 'How much stress do you feel in your daily life?'. We 
classified the responses of 'extremely high' and 'high' into the perceived stress group. If the participants answered 'yes' to 'Have you experienced a continuous feeling of sadness or despair for over 2 weeks that interfered with your daily life in the last year?' and 'Have you ever seriously considered committing suicide in the last year?', they were classified into the depressed and suicidal ideation groups, respectively. As depressive experience and suicidal ideation were surveyed only in 2012, 2013 and 2015, and experience of mental health counselling was surveyed using the same question only in 2012 and 2015, the analysis using these variables included only data from the surveyed year.

\section{Food insecurity}

The Korean food insecurity questionnaire was developed by the Korea Centers for Disease Control and Prevention based on an eighteen-item US Household Food Security Survey Module (US-HFSSM). The household food insecurity questionnaire consisted of three questions on household food conditions and seven questions for adults. The additional eight questions for children were administered to households with children. Each question specified the food condition due to lack of money to buy food 1 year before the interview. A detailed description concerning the development and validation of this index has been published elsewhere ${ }^{(29,30)}$. In brief, the Cronbach's $\alpha$ coefficient for internal consistency was 0.85 , ranging from 0.83 to 0.86 after eliminating each item. To classify the household food insecurity status, a score of 1 for affirmative responses indicated food-insecure conditions (e.g. 'often' or 'sometimes', 'almost every month' or 'some months but not every month' and 'yes') or a score of 0 for all other responses was given to each question, and the scores were summed. Based on the criteria of the US-HFSSM ${ }^{(1)}$, households were classified into food-secure and food-insecure groups if the total score was $<2$ and $\geq 3$, respectively. The status of food insecurity of all family members within the household was classified.

\section{Statistical analyses}

To consider the complex sampling design of the KNHANES, all data on food insecurity from the 20122015 KNHANES were pooled into one data set and new sampling weights were calculated by dividing the sampling weight assigned to the participant by the number of survey years. The new sampling weight was applied to all analyses. Data were presented as percentages with their standard errors. $P$ values for percentage differences between the food-secure and food-insecure groups were calculated using the $\chi^{2}$ for univariate analysis. Age- or multivariateadjusted prevalence, OR and $95 \%$ CI were calculated by multivariate logistic regression analysis after adjusting for confounding factors. Age- or multivariate-adjusted prevalence was calculated using the LSMEANS statement and the OM option for specifying the weighting scheme in the PROC SURVEYLOGISTIC statement. Confounding factors were selected based on their association with food insecurity or health outcomes in the present study or established risk factors for health outcomes. Multivariate models adjusted for age (continuous), household income (quartiles), place of residence (rural or urban), smoking (never, past or current smoker), alcohol drinking (none, <high-risk drinker or high-risk drinker), regular walking (yes or no) and BMI $\left(<18.5,18.5-24.9\right.$ or $\left.\geq 25.0 \mathrm{~kg} / \mathrm{m}^{2}\right)$. The statistical significance was defined as $P<0.05$. The statistical software package IBM SPSS Statistics version 23 was used to analyse all data from a complex sample survey.

\section{Results}

\section{General characteristics of men and women in the study}

The general characteristics of men and women are presented in Table 1 . The prevalence of men and women with food insecurity was 7.6 and $9.2 \%$, respectively. The proportions of older adults aged $\geq 65$ years and of individuals with a lower education level were higher in men and women with food insecurity than in those with food security. Some 54.0 and $53.2 \%$ of men and women with food insecurity, respectively, were reported to have the lowest household income. The proportion of divorced or widowed men and women with food insecurity was higher than in those without food insecurity. The proportions of recipients of basic livelihood security and food assistance were 6.0 and $2.2 \%$ in men, respectively, and 8.2 and $3.1 \%$ in women, respectively, showing a higher proportion in men and women with food insecurity.

\section{Prevalence of disease management-related factors according to food insecurity status}

Table 2 shows the prevalence of taking medications, trying to control weight, health examination, education or counselling related to disease, and unmet health-care needs according to food insecurity. The prevalence of taking medications to treat hypertension, diabetes and hypercholesterolaemia was not different in the food-insecure group after adjusting for confounding factors. The prevalence of trying to control weight in men with food insecurity $(68.8 \%)$ was higher than that in men with food security $(63.4 \%)$. Significant differences were found in mental health counselling and unmet health-care needs in men and women with food insecurity. Compared with adults with food security (1.1 and $3.3 \%$ in men and women with food security, respectively), men (4.2\%) and women $(5.9 \%)$ with food insecurity had a higher prevalence of counselling. The prevalence of unmet health-care needs was also higher in adults with food insecurity, accounting for 13.1 and $31.4 \%$ in men and women with food insecurity, respectively. 
Table 1 General characteristics of participants aged $\geq 30$ years $(n 17934)$ by food insecurity status, Korea National Health and Nutrition Examination Survey (KNHANES) 2012-2015

\begin{tabular}{|c|c|c|c|c|c|c|c|c|c|c|c|c|c|c|}
\hline \multirow[b]{3}{*}{ Characteristic } & \multicolumn{7}{|c|}{ Men } & \multicolumn{7}{|c|}{ Women } \\
\hline & \multicolumn{2}{|c|}{$\begin{array}{c}\text { Total } \\
(n 7247)\end{array}$} & \multicolumn{2}{|c|}{$\begin{array}{c}\text { Food } \\
\text { secure } \\
(n 6699)\end{array}$} & \multicolumn{2}{|c|}{$\begin{array}{c}\text { Food } \\
\text { insecure } \\
(n 548)\end{array}$} & \multirow[b]{2}{*}{$P$ value* } & \multicolumn{2}{|c|}{$\begin{array}{c}\text { Total } \\
(n 10687)\end{array}$} & \multicolumn{2}{|c|}{$\begin{array}{c}\text { Food } \\
\text { secure } \\
(n 9703)\end{array}$} & \multicolumn{2}{|c|}{$\begin{array}{c}\text { Food } \\
\text { insecure } \\
(n 984)\end{array}$} & \multirow[b]{2}{*}{$P$ value* } \\
\hline & $\%$ & SE & $\%$ & SE & $\%$ & SE & & $\%$ & SE & $\%$ & SE & $\%$ & SE & \\
\hline \multicolumn{15}{|l|}{ Age } \\
\hline 30-44 years & 38.9 & 0.9 & $39 \cdot 6$ & 0.9 & 29.5 & 2.5 & \multirow[t]{4}{*}{$<0.001$} & 35.5 & 0.7 & $36 \cdot 3$ & 0.7 & $27 \cdot 2$ & $2 \cdot 0$ & \multirow[t]{4}{*}{$<0.001$} \\
\hline $45-54$ years & 26.4 & 0.7 & $26 \cdot 1$ & 0.7 & $30 \cdot 8$ & 2.5 & & 24.8 & 0.5 & 24.7 & 0.6 & $26 \cdot 3$ & 1.8 & \\
\hline $55-64$ years & $17 \cdot 4$ & 0.5 & $17 \cdot 4$ & 0.5 & $17 \cdot 8$ & 1.8 & & $18 \cdot 4$ & 0.5 & $18 \cdot 6$ & 0.5 & $16 \cdot 6$ & 1.3 & \\
\hline$\geq 65$ years & $17 \cdot 3$ & 0.5 & $16 \cdot 9$ & 0.5 & 21.9 & 1.7 & & $21 \cdot 3$ & 0.5 & $20 \cdot 4$ & 0.5 & 29.9 & 1.7 & \\
\hline \multicolumn{15}{|l|}{ Household income } \\
\hline Q1 (lowest) & $25 \cdot 0$ & 0.7 & $22 \cdot 7$ & 0.7 & $54 \cdot 0$ & $2 \cdot 6$ & \multirow[t]{4}{*}{$<0.001$} & 24.8 & 0.6 & 21.9 & 0.6 & 53.2 & 1.9 & \multirow[t]{4}{*}{$<0.001$} \\
\hline Q2 & 25.4 & 0.7 & $25 \cdot 3$ & 0.7 & $25 \cdot 8$ & $2 \cdot 2$ & & $25 \cdot 0$ & 0.5 & 24.5 & 0.6 & $30 \cdot 1$ & 1.7 & \\
\hline Q3 & 24.9 & 0.6 & $25 \cdot 7$ & 0.7 & $13 \cdot 8$ & $2 \cdot 0$ & & $24 \cdot 8$ & 0.5 & $26 \cdot 1$ & 0.6 & $12 \cdot 2$ & 1.2 & \\
\hline Q4 (highest) & 24.8 & 0.8 & $26 \cdot 2$ & 0.8 & 6.4 & 1.4 & & $25 \cdot 3$ & 0.7 & 27.5 & 0.8 & 4.5 & 0.7 & \\
\hline \multicolumn{15}{|l|}{ Education level } \\
\hline$\leq$ Elementary school & $14 \cdot 0$ & 0.5 & $13 \cdot 0$ & 0.5 & $27 \cdot 4$ & $2 \cdot 2$ & \multirow[t]{4}{*}{$<0.001$} & 26.5 & 0.6 & $24 \cdot 8$ & 0.6 & $43 \cdot 8$ & $2 \cdot 0$ & \multirow[t]{4}{*}{$<0.001$} \\
\hline Middle school & $10 \cdot 3$ & 0.4 & 9.9 & 0.4 & 15.8 & $2 \cdot 1$ & & 11.2 & 0.4 & $11 \cdot 1$ & 0.4 & 12.4 & 1.3 & \\
\hline High school & $34 \cdot 1$ & 0.8 & $34 \cdot 1$ & 0.8 & 34.9 & $2 \cdot 6$ & & $33 . \overline{5}$ & 0.6 & 33.6 & 0.7 & $32 \cdot 0$ & $2 \cdot 0$ & \\
\hline$\geq$ College & 41.5 & 0.9 & $43 \cdot 0$ & 0.9 & $22 \cdot 0$ & $2 \cdot 4$ & & $28 \cdot 8$ & 0.7 & 30.5 & 0.8 & $11 \cdot 8$ & 1.3 & \\
\hline \multicolumn{15}{|l|}{ Marital status } \\
\hline Single & $10 \cdot 7$ & 0.5 & $10 \cdot 5$ & 0.5 & $12 \cdot 4$ & 1.9 & \multirow[t]{4}{*}{$<0.001$} & 4.4 & 0.3 & 4.5 & 0.3 & 3.5 & 0.8 & \multirow[t]{4}{*}{$<0.001$} \\
\hline Married & $83 \cdot 1$ & 0.6 & $84 \cdot 3$ & 0.6 & $68 \cdot 3$ & $2 \cdot 4$ & & $76 \cdot 0$ & 0.6 & $78 \cdot 1$ & 0.6 & $55 \cdot 0$ & $2 \cdot 0$ & \\
\hline Divorced or separated & 4.4 & 0.3 & 3.7 & 0.3 & 14.2 & 1.9 & & $5 \cdot 6$ & 0.3 & 4.5 & 0.3 & $16 \cdot 3$ & $1 \cdot 3$ & \\
\hline Widowed & 1.8 & 0.2 & 1.6 & 0.1 & $5 \cdot 2$ & 1.0 & & 14.0 & 0.4 & $12 \cdot 8$ & 0.4 & $25 \cdot 2$ & 1.7 & \\
\hline \multicolumn{15}{|l|}{ Area of residence } \\
\hline Urban & 79.5 & 1.5 & $79 \cdot 6$ & 1.5 & 78.9 & 2.6 & \multirow[t]{2}{*}{0.764} & $82 \cdot 2$ & 1.3 & 82.2 & 1.3 & $82 \cdot 7$ & 1.9 & \multirow{2}{*}{0.736} \\
\hline Rural & 20.5 & 1.5 & $20 \cdot 4$ & 1.5 & $21 \cdot 1$ & $2 \cdot 6$ & & $17 \cdot 8$ & 1.3 & $17 \cdot 8$ & 1.3 & $17 \cdot 3$ & 1.9 & \\
\hline Basic livelihood security & & & & & & & & & & & & & & \\
\hline Recipients & $6 \cdot 0$ & 0.4 & 4.7 & 0.4 & $22 \cdot 2$ & $2 \cdot 3$ & $<0.001$ & 8.2 & 0.5 & $6 \cdot 2$ & 0.4 & 27.9 & $2 \cdot 2$ & $<0.001$ \\
\hline No recipients & 94.0 & 0.4 & $95 \cdot 3$ & 0.4 & 77.8 & $2 \cdot 3$ & & $91 \cdot 8$ & 0.5 & 93.8 & 0.4 & $72 \cdot 1$ & $2 \cdot 2$ & \\
\hline Food assistance & & & & & & & & & & & & & & \\
\hline Recipients & $2 \cdot 2$ & 0.2 & 1.6 & 0.2 & $10 \cdot 2$ & 1.5 & $<0.001$ & $3 \cdot 1$ & 0.2 & $2 \cdot 2$ & 0.2 & $12 \cdot 1$ & 1.3 & $<0.001$ \\
\hline No recipients & $97 \cdot 8$ & 0.2 & $98 \cdot 4$ & 0.2 & $89 \cdot 8$ & 1.5 & & $96 \cdot 9$ & 0.2 & 97.8 & 0.2 & 87.9 & 1.3 & \\
\hline
\end{tabular}

All percentages and SE were calculated by applying sampling weights.

${ }^{*} P$ values for percentage differences between the two groups were calculated using the $\chi^{2}$ test.

tIncluding livelihood, housing, education, medical benefits, etc. from the government.

†Experience for the last 1 year of food assistance, including the NutriPlus ${ }^{+}$Program (a supplemental nutrition care programme for women, infants and children in Korea, adopting the US Special Supplemental Nutrition Program for Women, Infants, and Children (WIC)), senior meals programme in welfare centres or lunch-delivery programme for low-income families or children during school vacation.

\section{Association of physical and mental health with food insecurity status}

The association between food insecurity and physical and mental health is shown in Table 3. For physical health, the prevalence of obesity in women with food insecurity (37.4\%) was higher than in women with food security (30.7\%), but the prevalence of obesity was not different by food insecurity in men. On the contrary, the prevalence of underweight in men with food insecurity (5.9\%) was higher than in men with food security (3.0\%). No difference was found in hypertension, diabetes and hypercholesterolaemia prevalence in men and women with food insecurity. The OR for physical health outcomes were not significant in both men and women after adjusting for multivariate factors.

The risk of poor mental health was associated with food insecurity. The prevalence of adults with perceived stress, depressive experience and suicidal ideation was significantly higher in adults with food insecurity than in adults with food security. After adjusting for confounding factors, men and women with food insecurity showed significantly higher OR for mental health outcomes (OR $=1 \cdot 40,95 \% \mathrm{CI}$ $1 \cdot 06,1.85$ for perceived stress; OR $=1.79,95 \%$ CI 1.26 , 2.56 for depressive experience; $\mathrm{OR}=2.07,95 \% \mathrm{CI}$ $1.38,3.11$ for suicidal ideation in men; and $\mathrm{OR}=1 \cdot 80$, $95 \%$ CI $1.51,2.14$ for perceived stress; OR $=1.74$, $95 \%$ CI 1.38, 2.23 for depressive experience; and $\mathrm{OR}=2 \cdot 16,95 \% \mathrm{CI} 1 \cdot 66,2 \cdot 80$ for suicidal ideation in women) compared with men and women with food security.

\section{Combined effects of disease management and food insecurity status on physical and mental bealth}

The combined effect of disease management and food insecurity status on physical and mental health was 
Table 2 Prevalence of disease management-related factors by food insecurity status among participants aged $\geq 30$ years ( $n 17934$ ), Korea National Health and Nutrition Examination Survey (KNHANES) 2012-2015

\begin{tabular}{|c|c|c|c|c|c|c|c|c|c|c|}
\hline \multirow[b]{3}{*}{ Variable } & \multicolumn{5}{|c|}{ Men } & \multicolumn{5}{|c|}{ Women } \\
\hline & \multicolumn{2}{|c|}{$\begin{array}{l}\text { Food secure } \\
(n 6699) \\
\end{array}$} & \multicolumn{2}{|c|}{$\begin{array}{c}\text { Food } \\
\text { insecure } \\
(n 548)\end{array}$} & \multirow[b]{2}{*}{$P$ value* } & \multicolumn{2}{|c|}{$\begin{array}{l}\text { Food secure } \\
(n 9703)\end{array}$} & \multicolumn{2}{|c|}{$\begin{array}{c}\text { Food } \\
\text { insecure } \\
(n 984)\end{array}$} & \multirow[b]{2}{*}{$P$ value } \\
\hline & $\%$ & SE & $\%$ & SE & & $\%$ & SE & $\%$ & SE & \\
\hline \multicolumn{11}{|l|}{ Taking medications to treat diseases } \\
\hline \multicolumn{11}{|l|}{ Hypertension } \\
\hline Age-adjusted prevalence & 43.4 & 1.6 & $36 \cdot 7$ & 4.6 & $0 \cdot 164$ & $57 \cdot 4$ & 1.7 & $60 \cdot 7$ & $4 \cdot 1$ & 0.418 \\
\hline Multivariate-adjusted prevalence $\dagger$ & $42 \cdot 1$ & $2 \cdot 2$ & 37.8 & $5 \cdot 2$ & 0.406 & 51.9 & 3.0 & $56 \cdot 2$ & 4.9 & 0.353 \\
\hline \multicolumn{11}{|l|}{ Diabetes } \\
\hline Age-adjusted prevalence & $48 \cdot 1$ & $3 \cdot 1$ & $47 \cdot 7$ & $7 \cdot 3$ & 0.965 & $59 \cdot 0$ & $2 \cdot 7$ & $68 \cdot 1$ & $7 \cdot 1$ & 0.226 \\
\hline Multivariate-adjusted prevalence $\dagger$ & $52 \cdot 1$ & $4 \cdot 0$ & 49.7 & $8 \cdot 2$ & 0.760 & $44 \cdot 0$ & $5 \cdot 1$ & $55 \cdot 3$ & $10 \cdot 0$ & 0.194 \\
\hline \multicolumn{11}{|l|}{ Hypercholesterolaemia } \\
\hline Age-adjusted prevalence & 33.4 & $2 \cdot 2$ & 33.3 & $7 \cdot 3$ & 0.992 & 34.4 & 1.7 & 38.4 & $5 \cdot 0$ & 0.410 \\
\hline Multivariate-adjusted prevalence $\dagger$ & $29 \cdot 0$ & $3 \cdot 1$ & $30 \cdot 4$ & $7 \cdot 7$ & 0.848 & $30 \cdot 0$ & $2 \cdot 6$ & $36 \cdot 3$ & $5 \cdot 2$ & 0.170 \\
\hline \multicolumn{11}{|l|}{ Trying to control weight } \\
\hline Age-adjusted prevalence & 62.4 & 0.7 & $62 \cdot 2$ & $2 \cdot 4$ & 0.947 & $69 \cdot 0$ & 0.6 & $66 \cdot 8$ & 1.9 & 0.244 \\
\hline Multivariate-adjusted prevalence $\dagger$ & 63.4 & 1.0 & $68 \cdot 8$ & $2 \cdot 5$ & 0.038 & 69.9 & 0.9 & $69 \cdot 4$ & 1.9 & 0.792 \\
\hline \multicolumn{11}{|l|}{ Trying to lose weight } \\
\hline Ăge-adjusted prevalence & 34.5 & 0.7 & $30 \cdot 3$ & 2.5 & $0 \cdot 120$ & $45 \cdot 6$ & 0.6 & 40.5 & $2 \cdot 1$ & 0.022 \\
\hline Multivariate-adjusted prevalence $\dagger$ & $34 \cdot 8$ & 1.0 & $34 \cdot 4$ & $2 \cdot 9$ & 0.891 & 46.5 & 1.0 & $42 \cdot 8$ & $2 \cdot 2$ & 0.111 \\
\hline \multicolumn{11}{|l|}{ Trying to gain weight } \\
\hline Age-adjusted prevalence & $19 \cdot 9$ & 0.6 & 23.2 & $2 \cdot 3$ & 0.149 & $18 \cdot 7$ & 0.5 & 18.4 & 1.5 & 0.875 \\
\hline Multivariate-adjusted prevalence $\dagger$ & $20 \cdot 8$ & 0.8 & 28.0 & $2 \cdot 8$ & 0.005 & $17 \cdot 3$ & 0.7 & $17 \cdot 9$ & $1 \cdot 7$ & 0.709 \\
\hline \multicolumn{11}{|l|}{ Medical examination } \\
\hline Age-adjusted prevalence & $69 \cdot 2$ & 0.8 & $57 \cdot 6$ & $2 \cdot 7$ & $<0.001$ & $64 \cdot 0$ & 0.7 & $56 \cdot 7$ & 1.9 & $<0.001$ \\
\hline Multivariate-adjusted prevalence $\dagger$ & $69 \cdot 3$ & $1 \cdot 0$ & $65 \cdot 6$ & $2 \cdot 9$ & 0.183 & $60 \cdot 2$ & $1 \cdot 1$ & $58 \cdot 0$ & $2 \cdot 1$ & 0.325 \\
\hline \multicolumn{11}{|l|}{ Mental health counselling $\neq$} \\
\hline Age-adjusted prevalence & $1 \cdot 2$ & 0.2 & 5.4 & $2 \cdot 0$ & $<0.001$ & $3 \cdot 1$ & 0.3 & $7 \cdot 2$ & 1.4 & $<0.001$ \\
\hline Multivariate-adjusted prevalence $\dagger$ & $1 \cdot 1$ & 0.3 & $4 \cdot 2$ & 1.6 & 0.002 & $3 \cdot 3$ & 0.4 & 5.9 & $1 \cdot 2$ & 0.014 \\
\hline \multicolumn{11}{|l|}{ Nutrition education or counselling } \\
\hline Age-adjusted prevalence & 3.3 & 0.3 & $2 \cdot 5$ & 0.7 & 0.337 & 4.9 & 0.3 & 4.5 & 0.8 & 0.674 \\
\hline Multivariate-adjusted prevalence $\dagger$ & 3.7 & 0.4 & $3 \cdot 4$ & 1.0 & 0.831 & 4.4 & 0.4 & 3.7 & 0.8 & 0.465 \\
\hline \multicolumn{11}{|l|}{ Unmet health-care needs } \\
\hline Age-adjusted prevalence & $9 \cdot 0$ & 0.4 & $16 \cdot 1$ & $2 \cdot 0$ & $<0.001$ & $14 \cdot 8$ & 0.5 & 33.4 & 1.8 & $<0.001$ \\
\hline Multivariate-adjusted prevalence $\dagger$ & 8.2 & 0.6 & $13 \cdot 1$ & 1.9 & 0.002 & 15.5 & 0.7 & 31.4 & $2 \cdot 0$ & $<0.001$ \\
\hline
\end{tabular}

All percentages and SE were calculated by applying sampling weights.

${ }^{\star}$ Age-adjusted prevalence and $P$ values for percentage differences between the two groups were calculated using multivariate logistic regression analysis after adjusting for confounding variables.

†Adjusted for age (continuous), household income (quartiles), place of residence (rural or urban), smoking (never, past or current smoker), alcohol drinking (none, <high-risk drinker or high-risk drinker), regular walking (yes or no) and BMl $\left(<18.5,18.5-24.9\right.$ or $\left.\geq 25.0 \mathrm{~kg} / \mathrm{m}^{2}\right)$. BMl was excluded in the model for trying to lose weight.

¥Mental health counselling was surveyed using the same question only in 2012 and 2015 .

examined (Table 4). Men with food insecurity who could not use health-care services although needed were more likely to be obese or underweight compared with men with food security who could use these services $(\mathrm{OR}=2.03$, $95 \%$ CI $1.17,3.51$ for obesity; and OR $=3.34,95 \% \mathrm{CI}$ $1.02,10.9$ for underweight). Specifically, unmet health-care needs affected obesity differently in men with food security and insecurity $\left(P_{\text {interaction }}=0.029\right)$. Men with food insecurity who tried to gain weight had the highest prevalence and risk of underweight as compared with other adults who were food secure or did not try to gain weight $\left(\mathrm{OR}=3.48, \quad 95 \% \quad\right.$ CI $\left.1.25, \quad 9.70, \quad P_{\text {interaction }}=0.038\right)$. Women with food insecurity were more likely to be obese when they did not receive nutrition education or counselling compared with women with food security who received nutrition education or counselling $\left(P_{\text {interaction }}=0.010\right)$. In terms of mental health outcomes, adults with food insecurity who could not use health-care services although needed had the highest prevalence of all mental health problems than other adults who were food secure or could use health-care services and their health risk was about three times more than that in adults with food security who could use these services. In contrast, adults with food insecurity who received mental health counselling had the highest prevalence and risk of mental health problems than other adults who were food secure or did not receive mental health counselling (OR $=5 \cdot 45,95 \%$ CI $\left.2 \cdot 05,14 \cdot 5, P_{\text {interaction }}=0.028\right)$.

\section{Discussion}

The limited access to healthy foods and poor disease management are important in the risk of diseases. Adults with food insecurity have been known to be more likely to have barriers to disease management and a higher risk of chronic 
Table 3 Association of physical and mental health with food insecurity status among participants aged $\geq 30$ years $(n 17$ 934), Korea National Health and Nutrition Examination Survey (KNHANES) 2012-2015

\begin{tabular}{|c|c|c|c|c|c|c|c|c|c|c|}
\hline \multirow[b]{3}{*}{ Variable } & \multicolumn{5}{|c|}{ Men } & \multicolumn{5}{|c|}{ Women } \\
\hline & \multicolumn{2}{|c|}{$\begin{array}{l}\text { Food secure } \\
(n 6699)\end{array}$} & \multicolumn{2}{|c|}{$\begin{array}{l}\text { Food insecure } \\
(n 548)\end{array}$} & \multirow[b]{2}{*}{$P$ value* } & \multicolumn{2}{|c|}{$\begin{array}{l}\text { Food secure } \\
(n 9703)\end{array}$} & \multicolumn{2}{|c|}{$\begin{array}{l}\text { Food insecure } \\
\quad(n 984)\end{array}$} & \multirow[b]{2}{*}{$P$ value } \\
\hline & $\%$ or OR & SE or $95 \% \mathrm{Cl}$ & $\%$ or OR & SE or $95 \% \mathrm{Cl}$ & & $\%$ or OR & SE or $95 \% \mathrm{Cl}$ & $\%$ or OR & SE or $95 \% \mathrm{Cl}$ & \\
\hline \multicolumn{11}{|l|}{ Physical health } \\
\hline \multicolumn{11}{|l|}{ Obesity $\left(\geq 25 \cdot 0 \mathrm{~kg} / \mathrm{m}^{2}\right) \dagger$} \\
\hline Age-adjusted prevalence (\%) & 39.8 & 0.7 & $42 \cdot 0$ & $2 \cdot 5$ & \multirow[t]{3}{*}{0.416} & $30 \cdot 7$ & 0.6 & 37.4 & $1 \cdot 8$ & \multirow[t]{3}{*}{$<0.001$} \\
\hline Age-adjusted $\mathrm{OR}$ and $95 \% \mathrm{Cl}$ & 1.00 & Ref. & 1.10 & $0.88,1.36$ & & 1.00 & Ref. & 1.35 & $1.15,1.58$ & \\
\hline Multivariate-adjusted $\neq \mathrm{OR}$ and $95 \% \mathrm{Cl}$ & 1.00 & Ref. & 1.22 & $0.96,1.55$ & & 1.00 & Ref. & 1.13 & $0.94,1.36$ & \\
\hline \multicolumn{11}{|l|}{ Underweight $\left(<18.5 \mathrm{~kg} / \mathrm{m}^{2}\right) \dagger$} \\
\hline Age-adjusted prevalence (\%) & 3.0 & 0.4 & $5 \cdot 9$ & 1.3 & \multirow[t]{3}{*}{0.006} & $5 \cdot 1$ & 0.3 & $6 \cdot 0$ & 1.2 & \multirow[t]{3}{*}{0.373} \\
\hline Age-adjusted $\mathrm{OR}$ and $95 \% \mathrm{Cl}$ & 1.00 & Ref. & 2.01 & $1.23,3.29$ & & 1.00 & Ref. & 1.21 & $0.80,1.83$ & \\
\hline Multivariate-adjusted $\ddagger$ OR and $95 \% \mathrm{Cl}$ & 1.00 & Ref. & 1.50 & $0.83,2.73$ & & 1.00 & Ref. & 1.15 & $0.73,1.83$ & \\
\hline \multicolumn{11}{|l|}{ Hypertension§ } \\
\hline Age-adjusted prevalence (\%) & $31 \cdot 7$ & 0.8 & $35 \cdot 7$ & $2 \cdot 6$ & \multirow[t]{3}{*}{0.124} & 21.4 & 0.6 & $23 \cdot 3$ & 1.9 & \multirow[t]{3}{*}{0.310} \\
\hline Age-adjusted OR and $95 \% \mathrm{Cl}$ & 1.00 & Ref. & 1.20 & $0.95,1.51$ & & 1.00 & Ref. & 1.11 & $0.91,1.37$ & \\
\hline Multivariate-adjusted $\neq \mathrm{OR}$ and $95 \% \mathrm{Cl}$ & 1.00 & Ref. & $1 \cdot 20$ & $0.94,1.55$ & & 1.00 & Ref. & 1.04 & $0.84,1.29$ & \\
\hline \multicolumn{11}{|l|}{ Diabetes $\|$} \\
\hline Age-adjusted prevalence (\%) & $10 \cdot 7$ & 0.5 & $13 \cdot 0$ & $2 \cdot 0$ & \multirow[t]{3}{*}{$0 \cdot 215$} & $7 \cdot 3$ & 0.4 & 7.5 & 1.0 & \multirow[t]{3}{*}{0.825} \\
\hline Age-adjusted $\mathrm{OR}$ and $95 \% \mathrm{Cl}$ & 1.00 & Ref. & 1.25 & $0.88,1.77$ & & 1.00 & Ref. & 1.03 & $0.78,1.36$ & \\
\hline Multivariate-adjusted $\neq \mathrm{OR}$ and $95 \% \mathrm{Cl}$ & 1.00 & Ref. & 1.15 & $0.81,1.63$ & & 1.00 & Ref. & 0.91 & $0.68,1.22$ & \\
\hline \multicolumn{11}{|l|}{ Hypercholesterolaemiaף } \\
\hline Age-adjusted prevalence (\%) & 14.9 & 0.5 & 11.8 & 1.6 & \multirow[t]{3}{*}{0.098} & $17 \cdot 2$ & 0.5 & $16 \cdot 7$ & 1.4 & \multirow[t]{3}{*}{0.757} \\
\hline Age-adjusted OR and $95 \% \mathrm{Cl}$ & 1.00 & Ref. & 0.77 & $0.56,1.05$ & & 1.00 & Ref. & 0.97 & $0.78,1.20$ & \\
\hline Multivariate-adjusted $\neq$ OR and $95 \% \mathrm{Cl}$ & 1.00 & Ref. & 0.79 & $0.57,1.10$ & & 1.00 & Ref. & 0.94 & $0.75,1.19$ & \\
\hline Mental health & & & & & & & & & & \\
\hline Perceived stress & & & & & & & & & & \\
\hline Age-adjusted prevalence (\%) & 22.5 & 0.7 & $31 \cdot 8$ & $2 \cdot 8$ & $<0.001$ & $24 \cdot 0$ & 0.5 & 40.9 & 1.9 & $<0.001$ \\
\hline Age-adjusted OR and $95 \% \mathrm{Cl}$ & 1.00 & Ref. & 1.61 & $1 \cdot 24,2 \cdot 10$ & & 1.00 & Ref. & $2 \cdot 20$ & $1.87,2.58$ & \\
\hline Multivariate-adjusted $\neq \mathrm{OR}$ and $95 \% \mathrm{Cl}$ & 1.00 & Ref. & 1.40 & $1.06,1.85$ & & 1.00 & Ref. & 1.80 & $1.51,2.14$ & \\
\hline Depressive experience ${ }^{\star *}$ & & & & & & & & & & \\
\hline Áge-adjusted prevalence (\%) & $7 \cdot 2$ & 0.5 & $16 \cdot 4$ & $2 \cdot 1$ & $<0.001$ & $14 \cdot 0$ & 0.5 & 28.8 & $2 \cdot 2$ & $<0.001$ \\
\hline Age-adjusted $\mathrm{OR}$ and $95 \% \mathrm{Cl}$ & 1.00 & Ref. & 2.51 & $1.83,3.45$ & & 1.00 & Ref. & 2.48 & $1.99,3.11$ & \\
\hline Multivariate-adjusted $\ddagger$ OR and $95 \% \mathrm{Cl}$ & 1.00 & Ref. & 1.79 & $1.26,2.56$ & & 1.00 & Ref. & 1.74 & $1.38,2 \cdot 23$ & \\
\hline Suicidal ideation ${ }^{\star \star}$ & & & & & & & & & & \\
\hline Age-adjusted prevalence (\%) & $5 \cdot 2$ & 0.4 & 14.8 & $2 \cdot 2$ & $<0.001$ & $8 \cdot 1$ & 0.4 & $19 \cdot 8$ & & $<0.001$ \\
\hline Age-adjusted OR and $95 \% \mathrm{Cl}$ & 1.00 & Ref. & 3.15 & $2 \cdot 16,4.59$ & & 1.00 & Ref. & $2 \cdot 81$ & $2 \cdot 18,3.64$ & \\
\hline Multivariate-adjusted $\ddagger$ OR and $95 \% \mathrm{Cl}$ & 1.00 & Ref. & 2.07 & $1 \cdot 38,3 \cdot 11$ & & 1.00 & Ref. & $2 \cdot 16$ & $1 \cdot 66,2 \cdot 80$ & \\
\hline
\end{tabular}

Ref., reference category.

All percentages and SE, and $\mathrm{OR}$ and $95 \% \mathrm{Cl}$, were calculated by applying sampling weights.

*Age-adjusted prevalence and $P$ values for percentage differences between the two groups were calculated using multivariate logistic regression analysis after adjusting for age.

†The control group included individuals with a BMI of $18.5-24 \cdot 9 \mathrm{~kg} / \mathrm{m}^{2}$

+ Multivariate OR and $95 \%$ Cl were calculated by logistic regression analysis after adjusting for age (continuous), household income (quartiles), place of residence (rural or urban), smoking (never, past or current smoker), alcohol drinking (none, $<$ high-risk drinker or high-risk drinker), regular walking (yes or no), met medical service needs (yes or no), nutrition education or counselling (yes or no) and BMI (<18.5, 18.5-24.9 or $\geq 25.0 \mathrm{~kg} / \mathrm{m}^{2}$ ). Mental health counselling instead of nutrition education or counselling was included in the model of mental health. BMI was excluded in the model for obesity and underweight.

§Hypertension: $\geq 140 \mathrm{mmHg}$ for systolic blood pressure, $\geq 90 \mathrm{mmHg}$ for diastolic blood pressure, or user of anthypertensive agent.

Diabetes: $\geq 126 \mathrm{mg} / \mathrm{dl}$ for blood glucose, a previous diagnosis of diabetes by a physician, or user of hypoglycaemic agent or insulin.

IHypercholesterolemia: $\geq 240 \mathrm{mg} / \mathrm{dl}$ for blood total cholesterol or user of cholesterol-lowering agent. 
Table 4 Combined effects of disease management-related factors and food insecurity status on physical and mental health among participants aged $\geq 30$ years ( $n 17934)$, Korea National Health and Nutrition Examination Survey (KNHANES) 2012-2015

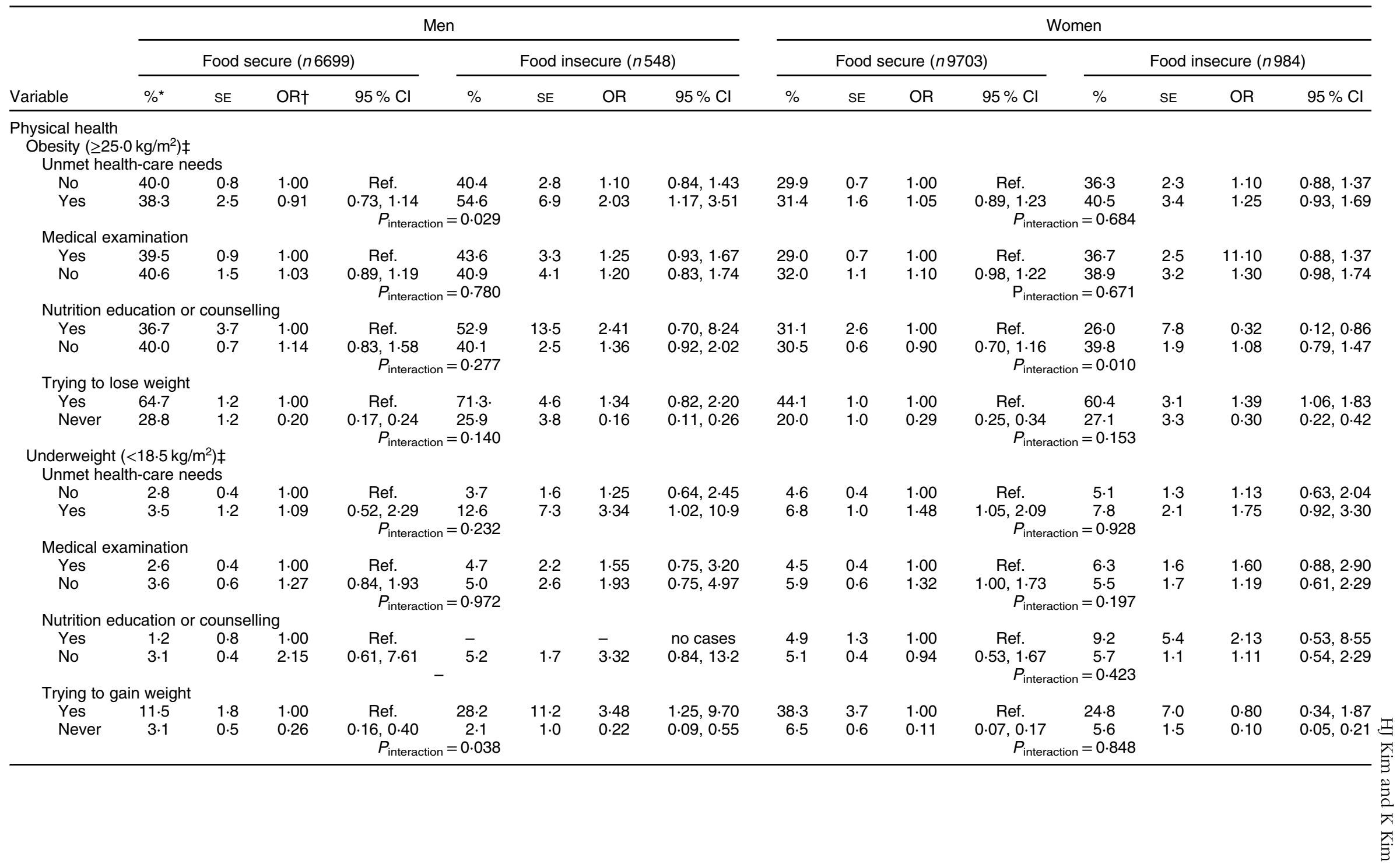




\section{Nublic Health Nutrition}

Table 4 Continued

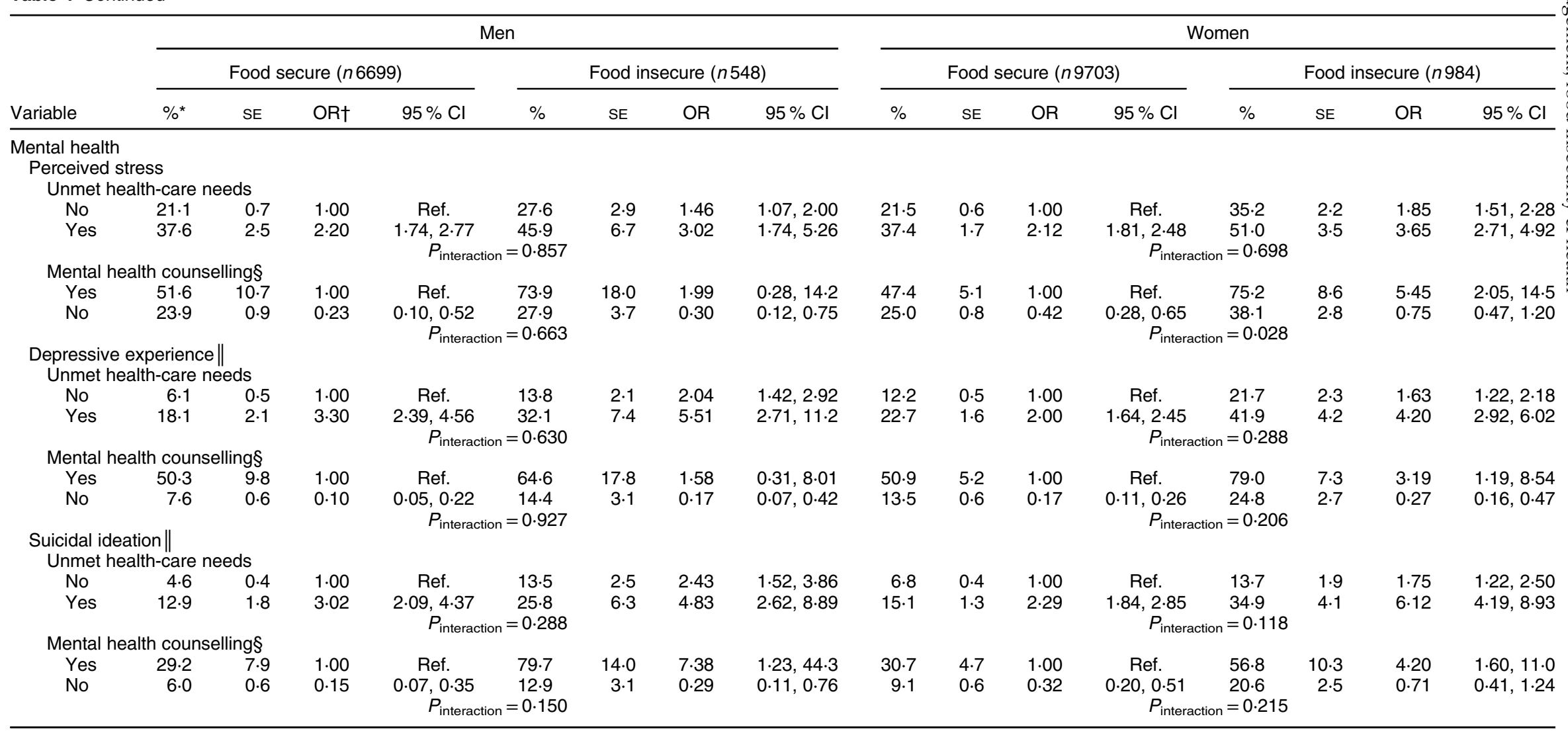

Ref., reference category.

*Age-adjusted prevalence was calculated by multivariate logistic regression analysis after adjusting for age.

† Multivariate $\mathrm{OR}$ and $95 \% \mathrm{Cl}$ were calculated by logistic regression analysis after adjusting for age (continuous), household income (quartiles), place of residence (rural or urban), smoking (never, past or current smoker), alcohol drinking (none, <high-risk drinker or high-risk drinker), regular walking (yes or no), met medical service needs (yes or no), nutrition education or counselling (yes or no) and BMI ( $<18.5,18.5-24.9$ or $\geq 25.0 \mathrm{~kg} / \mathrm{m}^{2}$ ). BMI was excluded in the model for obesity and underweight.

group included individuals with a BMI of $18.5-24.9 \mathrm{~kg} / \mathrm{m}^{2}$.

\$Mental health counselling was surveyed using the same question only in 2012 and 2015.

|Depressive experience and suicidal ideation were surveyed only in 2012, 2013 and 2015. 
diseases than adults with food security. Their passive disease management would exacerbate the risk of diseases, but studies on the combined effects of disease management and food insecurity on health outcomes are scarce. The present study found that among disease managementrelated factors, taking medications to treat diseases was not different by food insecurity status, but adults with food insecurity had higher unmet health-care needs and mental health counselling than adults with food security. The difference in disease management between adults with food security and food insecurity had a different impact on mental health and physical health outcomes.

Similar results have been shown in other studies in which food insecurity was associated with a lack of disease management and the two coexisting factors increased adverse effects on health ${ }^{(11-14,31,32)}$. People on low incomes with food insecurity have high rates of poor access to care and are more likely to delay care when needed, tending to result in increased risk of obesity and diabetes ${ }^{(14,32)}$. In the adult participants in the National Health Interview Survey, food-insecure people were less likely to use cost-related medication and suffered more from chronic conditions $^{(12,13)}$. Among adults with diabetes, food insecurity was associated with poor diabetes self-management and increased risk of hypoglycaemia ${ }^{(31)}$. On the other hand, a recent Canadian study reported that food insecurity was associated with increased health-care use and higher costs; the contrast with previous studies has been explained by different access to health insurance in the context of Canada's universal health-care system ${ }^{(33)}$.

The possible aetiological mechanism underlying the association between food insecurity and physical or mental health outcomes has been proposed in previous studies $^{(2,3,31,34)}$. Households with food insecurity could be more likely to experience episodic food shortages, thereby following an abnormal cyclic pattern of food consumption and metabolism alteration, and would be more dependent on inexpensive and high-energy-density foods. These negative experiences would also promote a stress response resulting in a preference for and intake of highly palatable foods that are energy dense and which can promote dietinduced obesity or insulin resistance ${ }^{(2,3,31,34)}$. In addition, the stress resulting from food shortages, constrained dietary options and complementary coping behaviours among food-insecure persons with low socio-economic status would result in psychological distress and poor mental health ${ }^{(6-8)}$. The pathways between food insecurity and health outcomes might be influenced by disease management $^{(11-14,31,32)}$. When food insecurity results in the development of chronic diseases, health-care expenditure and the time required for managing disease will increase. These circumstances put further strain on economic resources, exacerbating food insecurity. In this way, a cyclic relationship between food insecurity, poor health outcomes and disease management arises ${ }^{(34)}$. Thus, understanding the role of disease management in household food insecurity and the development of disease could be important for interventions targeting modifiable factors such as disease management.

In the present study, higher unmet health-care needs was an important risk factor for health problems in adults with food insecurity. Several studies have reported the major reasons for unmet health-care needs as financial burdens, limited availability of services or functional limitations in adults with food insecurity ${ }^{(12-15,35,36)}$. Additionally, a recent Korean study using Community Healthy Survey data found that use of health-care services to address mental health problems was positively associated with a high educational level ${ }^{(37)}$. The study explained that the reasons could be that higher educated adults could have more opportunities to obtain information on access to mental health services and less stigma of what others would think about receiving mental health services. The findings implied that an increased accessibility to mental health services and increased public awareness about the services are important for vulnerable populations, including adults with food insecurity.

Despite the importance of mental health counselling, ironically, adults with food insecurity who underwent mental health counselling were more likely to have mental health problems than those who did not undergo counselling. This unexpected result could be explained by reverse causal inference because of the cross-sectional nature of the data. Given that persistently needy individuals seek more help, an at-risk population with mental health problems might be more likely to undergo mental health counselling. The findings also reflect that the current services could not be adequate to manage these problems. Several studies reported that legal frameworks have been established to implement community-based mental health services, but professional staff and budget are lacking ${ }^{(38,39)}$. In addition, negative attitudes towards the services, distrust of the service effects, or lack of money and time could be the reasons for the non-persistent use of mental health services among the vulnerable population ${ }^{(40,41)}$. Therefore, for the low-income mental health high-risk group, the mental health service system might be improved first in terms of resource planning and management, as well as promotion to raise the awareness of services.

For physical health outcomes, the prevalence of obesity and underweight was different between adults with food security and insecurity, with a higher prevalence of underweight and obesity in men and women with food insecurity, respectively. The physical health outcomes were the worst in adults with food insecurity who did not meet health-care needs or did not receive nutrition education or counselling in the present study. In recent times, obesity is a major health issue in the disadvantaged population, particularly among children and women in Korea ${ }^{(10,42)}$. The disparity would be partially mediated by a lower intake of healthy foods, such as vegetables and fruits ${ }^{(18-21)}$. The present study showed that insufficient disease management, 
such as unmet health-care needs and low participation in nutrition education or counselling, could also affect the disparity of physical health problems. The health-care system should be more affordable and accessible to adults with food insecurity to reduce the inequality of the health-care burden which leads to health disparity.

Generally, in the present study, the gender difference in the relationship between food insecurity, disease management and health outcomes was not pronounced, suggesting that the potential influence of food insecurity and disease management on health is similar for men and women. Unexpectedly, both men and women were more likely to be obese or underweight regardless of food insecurity when they were trying to control weight compared with those who were not. This might be explained by reverse causality, like the result for the mental health counselling.

The present study has some limitations that should be addressed in future studies. First, the study was based on a cross-sectional survey; hence, only an association was observed and causality could not be confirmed clearly. Second, the mental health outcomes were assessed using a single-item self-reported questionnaire. In terms of the question for suicidal ideation, the single-item self-reported assessment had the possibility of increased risk of misclassification $^{(43)}$. On the other hand, several Western studies have found that a single-item self-reported question for depressive experience may be useful to identify depressive patients $^{(44-46)}$. However, one study among Korean older adults reported that a single question could substantially underestimate depression ${ }^{(47)}$. Therefore, the prevalence of mental health outcomes in the present study may be underestimated and further studies using questionnaires fully valid for mental health outcomes are necessary. Third, the status of household food insecurity was applied to individuals to measure food insecurity. There is a possibility that an individual's experience of food insecurity and that of family members are likely to differ depending on the allocation of resources within the household ${ }^{(1)}$. However, the study population is adult members who are likely to be similarly food insecure ${ }^{(1)}$, so that individual food insecurity measures applied from the household level measures might be suitable. Finally, we could not include possible confounding factors that were not available in the data set, such as substance use which could be an effect modifier of mental health in relation with food insecurity ${ }^{(48)}$. Despite several limitations, the strength of the present study is the use of a nationally representative sample showing that intervention in health-care management may require a policy to further reduce the inequality in health problems due to food insecurity.

\section{Conclusion}

In conclusion, adults with food insecurity had higher unmet health-care needs and mental health counselling than adults with food security. Unmet health-care needs was related increased risk of obesity for food-insecure men and mental health problems for both food-insecure men and women. In addition, lack of participation in nutrition education or counselling was related to increased risk of obesity for food-insecure women. Food insecurity is one of the most significant nutritional determinants of health status. The policy and programme for adults with food insecurity need to provide integrated and persistent health-care services with basic social services, such as food and housing, through the timely recognition and identification of the health-care needs of such adults with physical and mental health conditions.

\section{Acknowledgements}

Financial support: The funding for this study was provided by the National Research Foundation of Korea (NRF2016R1A2B4013798). The National Research Foundation of Korea had no role in the design, analysis or writing of this article. Conflict of interest: The authors declare no conflict of interest. Authorship: H.J.K. and K.K. conceived of the idea and designed the study; H.J.K. conducted the statistical analysis and wrote the method; K.K. wrote the draft of the manuscript; and both authors contributed to the final review of the manuscript. Etbics of human subject participation: All procedures and protocols used in the study were approved by the institutional review board of the Korea Centers for Disease Control and Prevention (2012-01EXP-01-2C, 2013-07CON-03-4C, 2013-12EXP03-5C, 2015-01-02-6C). Written informed consent regarding the survey was obtained from all participants.

\section{References}

1. Bickel G, Nord M, Price C et al. (2000) Measuring Food Security in the United States. Guide to Measuring Household Food Security. Alexandria, VA: Office of Analysis, Nutrition, and Evaluation, Food and Nutrition Service, US Department of Agriculture.

2. Laraia BA (2013) Food insecurity and chronic disease. Adv Nutr 4, 203-212.

3. Seligman HK, Laraia BA \& Kushel MB (2010) Food insecurity is associated with chronic disease among low-income NHANES participants. J Nutr 140, 304-310.

4. Jones AD (2017) Food insecurity and mental health status: a global analysis of 149 countries. Am J Prev Med 53, 264-273.

5. Weissman SK (2017) Comparing the physical and psychological effects of food security and food insecurity. Senior Honors Thesis, Eastern Michigan University.

6. Chung HK, Kim OY, Kwak SY et al. (2016) Household food insecurity is associated with adverse mental health indicators and lower quality of life among Koreans: results from the Korea National Health and Nutrition Examination Survey 2012-2013. Nutrients 8, 819.

7. Davison KM, Marshall-Fabien GL \& Tecson A (2015) Association of moderate and severe food insecurity with suicidal ideation in adults: national survey data from three Canadian provinces. Soc Psychiatry Psychiatr Epidemiol 50, 963-972. 
8. Davison KM \& Kaplan BJ (2015) Food insecurity in adults with mood disorders: prevalence estimates and associations with nutritional and psychological health. Ann Gen Psychiatry 14, 21.

9. Food Research \& Action Center (2015) Understanding the Connections: Food Insecurity and Obesity. Washington, DC: FRAC.

10. Chun IA, Ryu SY, Park J et al. (2015) Associations between food insecurity and healthy behaviors among Korean adults. Nutr Res Pract 9, 425-432.

11. Afulani P, Herman D, Coleman-Jensen A et al. (2015) Food insecurity and health outcomes among older adults: the role of cost-related medication underuse. J Nutr Gerontol Geriatr 34, 319-342.

12. Berkowitz SA, Seligman HK \& Choudhry NK (2014) Treat or eat: food insecurity, cost-related medication underuse, and unmet needs. Am J Med 127, 303-310.e3.

13. Herman D, Afulani P, Coleman-Jensen A et al. (2015) Food insecurity and cost-related medication underuse among nonelderly adults in a nationally representative sample. $A m J$ Public Health 105, e48-e59.

14. Kushel MB, Gupta R, Gee L et al. (2006) Housing instability and food insecurity as barriers to health care among lowincome Americans. J Gen Intern Med 21, 71-77.

15. Berkowitz SA, Meigs JB, DeWalt D et al. (2015) Material need insecurities, control of diabetes mellitus, and use of health care resources: results of the Measuring Economic Insecurity in Diabetes study. JAMA Intern Med 175, 257-265.

16. Ivers LC \& Cullen KA (2011) Food insecurity: special considerations for women. Am J Clin Nutr 94, issue 6, 1740S-1744S.

17. Kim HJ \& Oh K (2015) Household food insecurity and dietary intake in Korea: results from the 2012 Korea National Health and Nutrition Examination Survey. Public Health Nutr 18, 3317-3325.

18. Hong SA, Kim K \& Kim MK (2012) Trends in the inequality of fruit and vegetable consumption between education levels indicated by the Korea National Health and Nutrition Examination Surveys. Eur J Clin Nutr 66, 942-949.

19. Hong SA, Kim K \& Kim MK (2012) Educational attainment and differences in fruit and vegetable consumption among middle-aged adults in the Korean National Health and Nutrition Examination Survey IV. Nutr Res Pract 6, 263-269.

20. Kim K, Park SM \& Oh KW (2013) The impact of nutritional policy on socioeconomic disparity in the unhealthy food intake among Korean adolescents. Appetite 71, 388-395.

21. Kim K, Shin SC \& Shim JE (2015) Nutritional status of toddlers and preschoolers according to household income level: overweight tendency and micronutrient deficiencies. Nutr Res Pract 9, 547-553.

22. Ministry of Health and Welfare (2015) The National Health Plan 2020 (HP 2020). No. 11-1352000-000285-13. Sejong: The Ministry of Health and Welfare.

23. Steering Committee of the Western Pacific Region of the World Health Organization, International Association for the Study of Obesity \& International Obesity Task Force (2000) The Asia-Pacific Perspective: Redefining Obesity and Its Treatment. Melbourne, VIC: Health Communications Australia Pty Ltd.

24. US Department of Health and Human Services (2004) The Seventh Report of the Joint National Committee on Prevention, Detection, Evaluation, and Treatment of High Blood Pressure (JNC-7). Bethesda, MD: National Institutes of Health.

25. World Health Organization (2001) Definition, Diagnosis and Classification of Diabetes Mellitus and Its Complication. Geneva: WHO.

26. National Institutes of Health (2002) Third Report of the National Cholesterol Education Program (NCEP) Expert
Panel on Detection, Evaluation, and Treatment of High Blood Cholesterol in Adults (Adult Treatment Panel III). Bethesda, MD: National Institutes of Health.

27. Statistics Canada (2010) Canadian Community Health Survey. Annual Component - 2010 Questionnaire. Ottawa: Statistics Canada.

28. Statistics Canada (2011) Canadian Community Health Survey. Mental Health - Pilot Questionnaire. Ottawa: Statistics Canada.

29. Kim K, Hong SA, Kwon SO et al. (2011) Development of food security measures for the Korean National Health and Nutrition Examination Survey. Korean J Nutr 44, 551-561.

30. Kim K, Hong SA, Kwon SO et al. (2011) Validation of food security measures for the Korean National Health and Nutrition Examination Survey. Korean J Community Nutr 16, 771-781.

31. Seligman HK, Davis TC, Schillinger D et al. (2010) Food insecurity is associated with hypoglycemia and poor diabetes self-management in a low-income sample with diabetes. J Health Care Poor Underserved 21, 1227-1233.

32. Chan J, DeMelo M, Gingras J et al. (2015) Challenges of diabetes self-management in adults affected by food insecurity in a large urban centre of Ontario, Canada. Int J Endocrinol 2015, 903468

33. Tarasuk V, Cheng J, de Oliveira C et al. (2015) Association between household food insecurity and annual health care costs. CMAJ 187, E429-e436.

34. Seligman HK \& Schillinger D (2010) Hunger and socioeconomic disparities in chronic disease. $N$ Engl J Med 363, 6-9.

35. Kim YS, Lee J, Moon Y et al. (2018) Unmet healthcare needs of elderly people in Korea. BMC Geriatr 18, 98.

36. Srinivasan M \& Pooler JA (2018) Cost-related medication nonadherence for older adults participating in SNAP, 2013-2015. Am J Public Health 108, 224-230.

37. Jang J, Lee SA, Kim W et al. (2018) Factors associated with mental health consultation in South Korea. BMC Psychiatry 18, 17.

38. Jung JW, Che HR, Chun JY et al. (2012) Strengthening the Mental Health Care Systems for High Risk Groups. Report no. 2012-12. Sejong: Korea Institute for Health and Social Affairs.

39. You DK \& Lee YH (2018) A comparison of mental health service systems in South Korea and US: role of clinical psychologists in local community. Korean J Clin Psychol 37, 24-32.

40. Lee SH \& Song LE (2014) A Study on Policy for Supporting Mental Health of Women in Seoul. Seoul: Seoul Foundation of Women \& Family.

41. Lee SY (2012) Future directions and issues of mental health policies in Korea. Health Welfare Policy Forum 5, 68-77.

42. Yoon NH \& Kwon S (2014) The effects of community environmental factors on obesity among Korean adults: a multilevel analysis. Epidemiol Health 36, e2014036.

43. Millner AJ, Lee MD \& Nock MK (2015) Single-item measurement of suicidal behaviors: validity and consequences of misclassification. PLoS One 10, e0141606.

44. Reme SE \& Eriksen HR (2010) Is one question enough to screen for depression? Scand J Public Health 38, 618-624.

45. Watkins C, Daniels L, Jack C et al. (2001) Accuracy of a single question in screening for depression in a cohort of patients after stroke: comparative study. BMJ 323, 1159.

46. Maboney J, Drinka TK, Abler R et al. (1994) Screening for depression: single question versus GDS. J Am Geriatr Soc 42, 1006-1008.

47. Kim KD, Chun H, Kim IH et al. (2018) Validating a singlequestion depression measure among older adults. Int Psychogeriatr 30, 69-76.

48. Davison KM, Holloway C, Gondara L et al. (2018) Independent associations and effect modification between lifetime substance use and recent mood disorder diagnosis with household food insecurity. PLoS One 13, e0191072. 